\title{
Effects of Light Fields During Inflation
}

\author{
Takeshi Kobayashi ${ }^{1, *}$ and Shinji Mukohyama ${ }^{2}$, \\ 1 Institute for Cosmic Ray Research, The University of Tokyo, \\ 5-1-5 Kashiwanoha, Kashiwa, Chiba 277-8582, Japan \\ 2 Institute for the Physics and Mathematics of the Universe (IPMU), \\ The University of Tokyo, 5-1-5 Kashiwanoha, Kashiwa, Chiba 277-8583, Japan
}

\begin{abstract}
In the inflationary universe, there can be light fields other than the inflaton. We explore a possibility that such light fields source the primordial perturbations, while minimally affecting the inflaton dynamics. We show that during inflation, fluctuations of the light fields can be converted to adiabatic curvature perturbations, which accumulate and become significant by the end of the inflationary era. An additional goal of this work is to distinguish between light fields which can/cannot be ignored during inflation. Such criteria become useful for examining cosmological scenarios with multiple fields. As concrete examples, our results are applied to D-brane inflation models. We consider effects from KK modes (oscillation modes) of wrapped branes in monodromy-driven large-field models, and angular directions of throat geometries in warped D-brane inflation.
\end{abstract}

\section{INTRODUCTION}

The initial conditions for the Hot Big Bang cosmology can be set by having an exponential expansion phase in the early universe [1]. A simple way to drive such an inflationary stage is to have a scalar field, dubbed the inflaton, whose potential energy dominates the universe. However, microscopic descriptions of inflationary cosmology in many cases predict, or even require the existences of additional light fields during this era. One may expect that such light fields show up merely as tiny corrections to the inflaton action, having negligible influences on the inflaton dynamics. In this work, we show that even in such cases, light fields can leave significant imprints on the primordial curvature perturbations. We explore the possibility that light fields other than the inflaton generate the dominant contributions to the curvature perturbations while minimally affecting the inflaton dynamics. This may open up new possibilities for inflationary models in which the inflaton field cannot produce perturbations compatible with observational data.

An additional goal of this work is to distinguish between light fields which can and cannot be ignored during inflation. This is related to questions such as: When can we treat multi-field inflation models as single-field ones? What are the effects of unstabilized moduli during inflation? Implications of light fields on cosmology after inflation have been discussed extensively as the moduli problem [4] 6 , while in this paper we discuss the effects of light fields during inflation, especially on the generated curvature perturbations. We discuss conditions under which the existence of light fields can/cannot be ignored. This may have relevance to mechanisms such as curvaton [7-10] and modulated reheating [11, 12] scenarios which require (and often simply assume) light fields to have no effects during inflation.

How light fields generate curvature perturbations can simply be understood as follows: When a light field $\sigma$ shows up as corrections to the inflaton action, then the number of e-foldings $d \mathcal{N}$ obtained within a certain inflaton field range $d \phi$ also becomes dependent on $\sigma, d \mathcal{N}=F(\phi, \sigma) d \phi$. Therefore it is clear that fluctuations of $\sigma$ lead to inhomogeneous expansion of the universe, generating cosmological perturbations.

One can also consider the light field as one of the inflatons, and interpret the system as a multi-field inflation model. Then the perturbations can be understood as arising from patches of the universe taking different paths in field space, where the entropy perturbations are converted to curvature perturbations during inflation (see e.g. 13 17). We will see that such perturbations originating from the fluctuations of $\sigma$ can become dominant over the curvature perturbations from $\phi$.

As microscopic examples, our results are applied to D-brane inflation models in string theory. We discuss the model proposed in [18] where monodromy of wrapped branes allows large-field inflation. In this paper we further analyze effects of KK modes (oscillation modes) of the wrapped branes which can become light during inflation. We also comment on D-brane inflation in a warped throat geometry [19] with light angular directions.

The rest of the paper is divided as follows. We first study effects of light fields when they correct the inflaton action in the form (1) in Section II. Then in Section III, we further discuss cases where the light field corrections show up 
in different ways, as in (26) and (39). The results are applied to D-brane inflation models in Section IV] where the details of the calculations are shown in the appendix. We present our conclusions in Section $\mathrm{V}$

\section{INFLATION MODULATED BY LIGHT FIELDS}

In this paper we study implications of having additional light fields manifesting themselves as corrections to the inflaton action. For this purpose, let us start by considering the following toy Lagrangian,

$$
\frac{\mathcal{L}}{\sqrt{-g}}=-\frac{1}{2} g^{\mu \nu} \partial_{\mu} \phi \partial_{\nu} \phi\left(1-f \frac{\sigma^{2}}{\mu^{2-m} \phi^{m}}\right)-\frac{1}{2} g^{\mu \nu} \partial_{\mu} \sigma \partial_{\nu} \sigma-V(\phi)\left(1+g \frac{\sigma^{2}}{\mu^{2-m} \phi^{m}}\right)
$$

where the inflaton $(\phi)$ kinetic term and potential are modified by modulus $(\sigma)$ corrections. ( $\sigma$ can also be called the inflaton, but for convenience we call $\phi$ the inflaton and $\sigma$ the modulus in this paper.) Though we have omitted the Einstein-Hilbert term, we are assuming Einstein gravity. The modulus corrections are suppressed by some mass scale $\mu$, and the field value of the inflaton $\phi$ can also suppress the corrections during inflation. $f$ and $g$ are dimensionless constants. Since we consider cases where the modulus corrections are small, correction terms that are higher order in $\sigma$ are neglected. Lagrangians of this type show up in many cases, e.g., when having nonminimally coupled fields in the Jordan frame, from nonminimal Kähler potentials in supersymmetric models. Later in Section IV] we give explicit examples in the context of D-brane inflation models, where the moduli corrections are suppressed by superPlanckian $\phi$ (Subsection IVA) or by approximate symmetries (Subsection IV B), thus allowing the moduli to be light during inflation. One may encounter microscopic models involving more complicated moduli corrections, but the crucial points can be captured by studying the above Lagrangian. Cases where the $\sigma$-corrections to the inflaton kinetic term and potential have different minima are later studied in Subsection IIIB.

We discuss curvature perturbations arising from fluctuations $\delta \phi$ and $\delta \sigma$. Since we focus on cases where the $\sigma$ corrections to the inflaton action are small, $\delta \phi$ almost directly generates adiabatic curvature perturbations at the time when the fluctuations exit the horizon. On the other hand, $\delta \sigma$ mainly produces entropy perturbations which can be converted to curvature perturbations during inflation. This kind of curvature perturbations arise due to $\delta \sigma$ kicking patches of the universe to different classical trajectories in the $\phi-\sigma$ plane, generating perturbations in the number of e-foldings $\delta \mathcal{N}$ among different trajectories. As we will soon see, when the entropy perturbations from $\delta \sigma$ are sufficiently transformed to adiabatic ones, then $\partial \mathcal{N} / \partial \sigma$ becomes dominant over $\partial \mathcal{N} / \partial \phi$.

The modulus correction to the inflaton kinetic term (i.e. the term with the coefficient $f$ in (1)) modifies the inflaton velocity, whereas the correction to the inflaton potential (i.e. the term with $g$ ) modifies the inflaton velocity as well as the Hubble parameter during inflation, cf. (11) and (12). Therefore inflation proceeds differently among different trajectories in the $\phi-\sigma$ plane. $\delta \mathcal{N}$ produced in this way is amplified when the trajectories during inflation diverge and differences among patches expand. On the other hand, in cases where the trajectories converge, the later transformation of the entropy to adiabatic perturbations is suppressed. ${ }^{1}$ In this paper, no matter the trajectories diverge or converge during inflation, we assume that after the inflationary era the trajectories converge to a single one (as is the case for the examples in Section IV).

Now let us actually compute $\delta \mathcal{N}$ produced from $\delta \phi$ and $\delta \sigma$, and see under which conditions the curvature perturbations sourced by the modulus field become significant.

In a flat FRW universe, the equations of motion for $\phi$ and $\sigma$, and the Friedmann equation are

$$
\begin{gathered}
\ddot{\phi}\left(1-f \frac{\sigma^{2}}{\mu^{2-m} \phi^{m}}\right)+3 H \dot{\phi}\left\{1-\left(1+\frac{2}{3} \frac{\dot{\sigma}}{H \sigma}-\frac{m}{6} \frac{\dot{\phi}}{H \phi}\right) f \frac{\sigma^{2}}{\mu^{2-m} \phi^{m}}\right\}=-V^{\prime}\left\{1+\left(1-\frac{m V}{V^{\prime} \phi}\right) g \frac{\sigma^{2}}{\mu^{2-m} \phi^{m}}\right\}, \\
\ddot{\sigma}+3 H \dot{\sigma}=-\left(2 g V+f \dot{\phi}^{2}\right) \frac{\sigma}{\mu^{2-m} \phi^{m}} \\
3 H^{2} M_{p}^{2}=\frac{1}{2} \dot{\phi}^{2}\left(1-f \frac{\sigma^{2}}{\mu^{2-m} \phi^{m}}\right)+\frac{1}{2} \dot{\sigma}^{2}+V\left(1+g \frac{\sigma^{2}}{\mu^{2-m} \phi^{m}}\right),
\end{gathered}
$$

\footnotetext{
${ }^{1}$ More precisely, distances among trajectories in field space do not necessarily reflect how differently inflation proceeds among patches. One should keep in mind that the diverging/converging discussion in the text is adopted for illustrative purposes.
} 
where derivatives with respect to $\phi$ are denoted by primes, and time $t$ derivatives by overdots.

We assume in this paper that $\phi$ is under slow-roll inflation, though estimations can be carried out in a similar manner with inflation models giving different inflaton dynamics, e.g. rapid-roll [20] 22], Dirac-Born-Infeld (DBI) inflation models [23, 24]. Also, we focus on cases where the $\sigma$-corrections to the inflationary dynamics are tiny, therefore, (2) and (4) are approximated by

$$
3 H \dot{\phi} \sim-V^{\prime}, \quad 3 H^{2} M p^{2} \sim V .
$$

We further assume

$$
|g| \gtrsim|f|
$$

(cases with $g=0$ are discussed in Subsection III A), and that $\sigma$ is slow-rolling under the approximation

$$
3 H \dot{\sigma} \sim-2 g V \frac{\sigma}{\mu^{2-m} \phi^{m}}
$$

Then one can check that the necessary conditions for the approximations (5) and (7) to hold are the absolute values of the following quantities be sufficiently smaller than one,

$$
\begin{aligned}
& \epsilon \equiv \frac{M_{p}^{2}}{2}\left(\frac{V^{\prime}}{V}\right)^{2}, \quad \eta \equiv M_{p}^{2} \frac{V^{\prime \prime}}{V}, \\
& g \frac{\sigma^{2}}{\mu^{2-m} \phi^{m}}, \quad \frac{m V}{V^{\prime} \phi} g \frac{\sigma^{2}}{\mu^{2-m} \phi^{m}}, \\
& m \frac{\dot{\phi}}{H \phi} \sim-m \frac{M_{p}^{2} V^{\prime}}{\phi V}, \quad g \frac{M_{p}^{2}}{\mu^{2-m} \phi^{m}},
\end{aligned}
$$

where roughly speaking, the first line is the slow-roll conditions for $\phi$, the second line for the modulus corrections to be minimal, and the third line for slow-rolling of $\sigma$. The smallness of the last quantity in the third line is equivalent to the effective mass of $\sigma$ being smaller than the Hubble parameter, i.e. $m_{\sigma}^{2} \ll H^{2}$.

To make the discussion concrete, henceforth we consider large-field inflation with

$$
V(\phi) \propto \phi^{n}, \quad \text { where } \quad n=\mathcal{O}(1) .
$$

Computations with other inflaton potentials are straightforward, though we expect results obtained from $(9)$ to be rather general. Implications for other potentials including small-field models will be briefly discussed at the end of the section. Then, given $|m| \leq \mathcal{O}(1)$, the condition $(8)$ is translated to

$$
\frac{M_{p}^{2}}{\phi^{2}} \ll 1, \quad\left|g \frac{\sigma^{2}}{\mu^{2-m} \phi^{m}}\right| \ll 1, \quad\left|g \frac{M_{p}^{2}}{\mu^{2-m} \phi^{m}}\right| \ll 1 .
$$

Now we need approximations more precise than (5) for the inflaton dynamics. Dropping terms in (2) and (4) that are clearly smaller than order $g \frac{\sigma^{2}}{\mu^{2-m} \phi^{m}}$ corrections to $(5)$, one can write down

$$
\begin{gathered}
\ddot{\phi}+3 H \dot{\phi}\left(1-f \frac{\sigma^{2}}{\mu^{2-m} \phi^{m}}\right) \simeq-V^{\prime}\left\{1+\left(1-\frac{m}{n}\right) g \frac{\sigma^{2}}{\mu^{2-m} \phi^{m}}\right\}, \\
3 H^{2} M_{p}^{2} \simeq \frac{1}{2} \dot{\phi}^{2}+V\left(1+g \frac{\sigma^{2}}{\mu^{2-m} \phi^{m}}\right) .
\end{gathered}
$$

Hence the number of e-foldings $d \mathcal{N}=d a / a$ is given by

$$
d \mathcal{N}=H d t=H \frac{d \phi}{\dot{\phi}} \simeq-\frac{V d \phi}{M_{p}^{2} V^{\prime}}\left\{1+\left(\frac{m}{n} g-f\right) \frac{\sigma^{2}}{\mu^{2-m} \phi^{m}}+\mathcal{O}(\epsilon, \eta)\right\},
$$

where we have expressed terms $\frac{\dot{\phi}^{2}}{V} \times \mathcal{O}(1), \frac{\ddot{\phi}}{V^{\prime \prime}} \times \mathcal{O}(1)$ as $\mathcal{O}(\epsilon, \eta)$. One should note that unless $m \neq 0$, the modulus correction to the inflaton potential (i.e. the term with $g$ ) is canceled between (11) and (12), and leaves no effect at linear order in (13). Now, (5) and (7) give

$$
\frac{d \sigma}{d \phi}=\frac{\dot{\sigma}}{\dot{\phi}} \sim 2 g \frac{V}{V^{\prime}} \frac{\sigma}{\mu^{2-m} \phi^{m}}
$$


which is integrated to yield

$$
\ln \frac{\sigma}{\sigma_{*}} \sim\left\{\begin{array}{cc}
\frac{2 g}{n} \ln \frac{\phi}{\phi_{*}} & (m=2) \\
\frac{2 g}{n(2-m)} \frac{\phi^{2-m}-\phi_{*}^{2-m}}{\mu^{2-m}} & (m \neq 2)
\end{array}\right.
$$

where $\sigma_{*}$ and $\phi_{*}$ are their field values at some fixed time. Substituting (15) into (13) yields an expression of the form $d \mathcal{N}=F\left(\phi, \phi_{*}, \sigma_{*}\right) d \phi$. (However, note that the errors of the leading order approximation can be amplified when taking the exponential of both sides of $[15)$ before substitution. |(terms in $(10)) \times(15) \mid \ll 1$ is required for good approximation.) By changing the variables $\left(\phi, \phi_{*}, \sigma_{*}\right) \rightarrow(\tilde{\phi}, \phi, \sigma)$, the number of e-foldings obtained from a point $(\phi, \sigma)$ in field space to the end of inflation is expressed as

$$
\mathcal{N}(\phi, \sigma)=\int_{\phi}^{\phi_{f}} F(\tilde{\phi}, \phi, \sigma) d \tilde{\phi}
$$

where $\phi_{f}$ is the value of $\phi$ at the end of inflation. Then by using the $\delta \mathcal{N}$-formalism [25 28], the curvature perturbation is given by

$$
\delta \mathcal{N}=\mathcal{N}(\phi+\delta \phi, \sigma+\delta \sigma)-\mathcal{N}(\phi, \sigma)=\frac{\partial \mathcal{N}}{\partial \phi} \delta \phi+\frac{\partial \mathcal{N}}{\partial \sigma} \delta \sigma+\cdots
$$

To leading order, the number of e-foldings obtained from $(\phi, \sigma)$ is

$$
\mathcal{N} \sim \frac{\phi^{2}-\phi_{f}^{2}}{2 n M_{p}^{2}}
$$

Since the $\sigma$-corrections are small, the surface where inflation ends in field space is almost independent of the modulus field. Therefore let us take $\phi_{f}$ as a constant for the moment. Later on, we justify this assumption. Then, the part of $\delta \mathcal{N}$ linear in $\delta \phi$ is given by

$$
\frac{\partial \mathcal{N}}{\partial \phi} \sim \frac{V}{M_{p}^{2} V^{\prime}}=\frac{\phi}{n M_{p}^{2}}
$$

This familiar result represents adiabatic curvature perturbations generated due to $\delta \phi$ pushing patches of the universe along the classical trajectories in the $\phi-\sigma$ plane. $^{2}$

The leading contribution for $\partial \mathcal{N} / \partial \sigma$ comes from the second term in the curly brackets in (13). (Note that the $\mathcal{O}(\epsilon, \eta)$ terms may be larger than the second term, but their dependence on $\sigma$ are schematically of the form $\epsilon=$ $\epsilon_{0}\left(1+\mathcal{O}\left(g \frac{\sigma^{2}}{\mu^{2-m} \phi^{m}}\right)\right)$ with $\epsilon_{0}$ the value in the absence of modulus corrections, hence their contributions to $\partial \mathcal{N} / \partial \sigma$ are suppressed by $\mathcal{O}(\epsilon, \eta)$.) Thus we obtain

$$
\begin{aligned}
& \frac{\partial \mathcal{N}}{\partial \sigma} \sim \begin{cases}\frac{1}{2}\left(\frac{2}{n}-\frac{f}{g}\right) \frac{\sigma}{M_{p}^{2}}\left\{1-\left(\frac{\phi_{f}}{\phi}\right)^{\frac{4}{n} g}\right\} & (m=2) \\
\frac{1}{2}\left(\frac{m}{n}-\frac{f}{g}\right) \frac{\sigma}{M_{p}^{2}}\left\{1-\exp \left(\frac{4 g}{n(2-m)} \frac{\phi_{f}^{2-m}-\phi^{2-m}}{\mu^{2-m}}\right)\right\} & (m \neq 2)\end{cases} \\
& \sim \frac{1}{2}\left(\frac{m}{n}-\frac{f}{g}\right) \frac{\sigma}{M_{p}^{2}}\left\{1-\left(\frac{\sigma_{f}}{\sigma}\right)^{2}\right\} \text {. }
\end{aligned}
$$

Note that we have used $\sqrt{15}$ in moving to the last line. ${ }^{3}$ This shows that when $\sigma$ has a positive effective mass squared (i.e. $g>0$, provided $\phi$ and $\mu$ being positive) and $\sigma$ rolls towards zero, then $\partial \mathcal{N} / \partial \sigma \propto \sigma / M_{p}^{2}$. On the other

\footnotetext{
${ }^{2}$ Contribution to $\frac{\partial \mathcal{N}}{\partial \phi}$ from the $\frac{\sigma^{2}}{\mu^{2-m} \phi^{m}}$ term in 13 represents differences among trajectories, similar to $\frac{\partial \mathcal{N}}{\partial \sigma}$. This, compared to 19 , is suppressed by $g \frac{\sigma_{f}^{2}}{\mu^{2-m} \phi^{m}}$. This factor can come close to or exceed unity only under $m<0$ and $\sigma_{f}^{2} \gg M_{p}^{2}$.

${ }^{3}$ When the $\sigma$-corrections are independent of $\phi$, i.e. when $m=0$, our results 19 and 20 can be checked to agree with the result (3.24) in [14] where generic two-field inflation models with separable potentials are studied.
} 
hand, when the modulus has a tachyonic mass (i.e. $g<0$ ) and $|\sigma|$ grows, then adjacent trajectories diverge towards the end of inflation and curvature perturbations are amplified by the factor $-\left(\sigma_{f} / \sigma\right)^{2}$. Major conversion of entropy perturbations from $\delta \sigma$ to adiabatic curvature perturbations $\delta \mathcal{N}$ occurs at early (later) times for converging (diverging) trajectories.

The ratio between linear contributions to $\delta \mathcal{N}$ from $\delta \phi \sqrt{19}$ and $\delta \sigma(20)$ takes the form,

$$
\frac{\partial \mathcal{N} / \partial \sigma}{\partial \mathcal{N} / \partial \phi}=\mathcal{O}(1) \times \frac{\sigma}{\phi}\left\{1-\left(\frac{\sigma_{f}}{\sigma}\right)^{2}\right\} .
$$

Especially under $|\sigma|<|\phi|$ which is expected to hold for most microscopic models (e.g., Section IV), then curvature perturbations from $\sigma$ can become dominant over that from $\phi$ only when the modulus has a tachyonic mass. It is important to bear in mind that even when the absolute field value of $\sigma$ is always smaller than that of $\phi$, a large growth of $|\sigma|$ such that $\left|\frac{\sigma}{\phi}\left(\frac{\sigma_{f}}{\sigma}\right)^{2}\right| \gtrsim 1$ makes contributions from $\delta \sigma$ significant. (However one should also note that this "amplification factor" $\left(\sigma_{f} / \sigma\right)^{2}$ is determined by $\phi$ and $\phi_{f}$, cf. 20 .)

Before turning to properties of curvature perturbations from $\delta \sigma$, let us briefly discuss possible corrections to the above formulae. First of all, where inflation ends (which is roughly when the slow-roll parameters become of order unity, i.e. $\left.\phi_{f}=\mathcal{O}(1) \times M_{p}\right)$ is not a constant but is slightly shifted by the modulus corrections. This can be written schematically in the form $\phi_{f}=\phi_{f_{0}}\left(1+\mathcal{O}\left(g \frac{\sigma_{f}^{2}}{\mu^{2-m} \phi_{f}^{m}}\right)\right)$ with $\phi_{f_{0}}$ the value of $\phi_{f}$ in the absence of the $\sigma$ corrections. Hence fluctuations of $\phi$ and $\sigma$ can modulate $\phi_{f}$, and curvature perturbations can be generated at the end of inflation [29, 30]. Furthermore, we should also note that different trajectories in the $\phi-\sigma$ plane end inflation at different energy densities. This starts the subsequent evolution of the universe from different temperatures, leading to extra $\delta \mathcal{N}$ after inflation (see, e.g. [17]). However, one can check that as long as 10 holds during inflation, both types of corrections are subdominant, i.e., such corrections sourced by $\delta \phi$ and $\delta \sigma$ are negligible compared to 19 and (20), respectively. ${ }^{4}$ We demonstrate the calculations only for the case we study in Subsection III A, but extending the estimations there $([35),(36))$ to the present case is straightforward.

We should also remark that in actual cases, the conditions (10) may break down during inflation. Inflation approaches an end when $M_{p}^{2} / \phi^{2} \rightarrow \mathcal{O}(1)$, but can also be terminated by the $\sigma$-correction $\left|g \frac{\sigma^{2}}{\mu^{2-m} \phi^{m}}\right|$ approaching unity. Meanwhile, inflation is not directly affected by $\left|g \frac{M_{p}^{2}}{\mu^{2-m} \phi^{m}}\right| \rightarrow \mathcal{O}(1)$, but this will accelerate/decelerate $\sigma$ and then the approximation (7) becomes invalid. Here, we emphasize that when $\sigma^{2} \gg \sigma_{f}^{2}$, i.e. the trajectories converge, most of the conversion of entropy to curvature perturbations occur at early times, hence the breakdown of (7) at later times does not affect the result 20 significantly. ${ }^{5}$ But when $\sigma^{2} \ll \sigma_{f}^{2}$, $\delta \mathcal{N}$ is generated substantially towards the end of inflation and 10 is required all through in order to trust 20 . is

Now let us focus on the curvature perturbations 20 generated by $\sigma$. By using $\mathcal{P}_{\delta \sigma}^{1 / 2}=H / 2 \pi$, the power spectrum

$$
\mathcal{P}_{\zeta_{\sigma}}=\left.\left(\frac{\partial \mathcal{N}}{\partial \sigma}\right)^{2}\left(\frac{H}{2 \pi}\right)^{2}\right|_{k=a H}
$$

where the right hand side is to be estimated at the time of horizon crossing. Then its spectral index can be computed,

$$
n_{s}-1=\frac{d \ln \mathcal{P}_{\zeta_{\sigma}}}{d \ln k} \sim \frac{\sigma_{f}^{2}+\sigma^{2}}{\sigma_{f}^{2}-\sigma^{2}} 4 g \frac{M_{p}^{2}}{\mu^{2-m} \phi^{m}}-n^{2} \frac{M_{p}^{2}}{\phi^{2}}
$$

where one should recall that $\phi_{f}$ is fixed. In addition, non-Gaussianity is sourced by the second derivative of $\mathcal{N}$ with respect to $\sigma$,

$$
\frac{\partial^{2} \mathcal{N}}{\partial \sigma^{2}} \sim \frac{1}{2}\left(\frac{m}{n}-\frac{f}{g}\right) \frac{1}{M_{p}^{2}}\left\{1-\left(\frac{\sigma_{f}}{\sigma}\right)^{2}\right\}
$$

\footnotetext{
4 To be precise, only when $\sigma \sim \sigma_{f}$, the additional $\delta \mathcal{N}$ sourced from $\delta \sigma$ can become comparable to 20. However in such cases, curvature perturbations from $\delta \sigma$ are suppressed anyway (cf. 21 ), and are negligible unless $\sigma^{2} \gg \phi^{2}$.

${ }^{5}$ However, as discussed above, when 10 breaks down then additional $\delta \mathcal{N}$ produced at the end and after inflation may become nonnegligible.
} 
Defining the non-Gaussianity parameter simply as the ratio between the three-point function and the squared twopoint function, we arrive at

$$
f_{\mathrm{NL}}=\frac{\left\langle\delta \mathcal{N}^{3}\right\rangle}{\left\langle\delta \mathcal{N}^{2}\right\rangle^{2}}=\frac{1}{2} \frac{\partial^{2} \mathcal{N} / \partial \sigma^{2}}{(\partial \mathcal{N} / \partial \sigma)^{2}} \sim\left(\frac{m}{n}-\frac{f}{g}\right)^{-1} \frac{M_{p}^{2}}{\sigma^{2}-\sigma_{f}^{2}} .
$$

One clearly sees that when $\sigma_{f}^{2} \gg \sigma^{2}$ (which is required for $\sigma$ with small field values to generate significant curvature perturbations), then $f_{\mathrm{NL}} \propto M_{p}^{2} / \sigma_{f}^{2}$ and a sub-Planckian modulus field produces large non-Gaussianity.

Before ending this section, let us pause to note implications for inflation models other than with $V(\phi) \propto \phi^{n}$. Given other inflaton potentials, (14) is integrated to yield results different from (15), leading to different growing or damping rate of $|\sigma|$. If then the growing rate of a tachyonic $\sigma$ is large, as we emphasized in the discussions above, $\partial \mathcal{N} / \partial \sigma$ is significantly amplified. We also mention that for small-field (i.e. $|\phi| \ll M_{p}$ ) slow-roll models, $\left|\frac{V}{V^{\prime} \phi}\right|=\frac{1}{\sqrt{2 \epsilon}}\left|\frac{M_{p}}{\phi}\right|$ is always larger than unity (in contrast to when $V(\phi) \propto \phi^{n}$ where $=\mathcal{O}(1)$ ). Then for $m \neq 0$ cases, the leading modulation to the inflaton dynamics will be the $\frac{m V}{V^{\prime} \phi} g \frac{\sigma^{2}}{\mu^{2-m} \phi^{m}}$ term in the right hand side of 2 .

\section{CASES WITH OTHER TYPES OF MODULUS CORRECTIONS}

In the previous section we have seen that light moduli with small field values can leave their imprints on primordial curvature perturbations only when they receive tachyonic backreaction and their field values are amplified. However, this is not necessarily the case when the modulus corrections enter the inflaton action in different manners. The two interesting cases we study in this section are when the $\sigma$-correction only enters the inflaton kinetic term, and when the $\sigma$-corrections in the inflaton kinetic term and potential have different minima.

\section{A. Modulating only the inflaton kinetic term}

First we study the case where the modulus correction only enters the inflaton kinetic term, i.e. $g=0$ in (1). Here $\sigma$ modulates inflation without receiving significant backreaction.

For simplicity, we fix $m$ in (1) to zero and study the Lagrangian

$$
\frac{\mathcal{L}}{\sqrt{-g}}=-\frac{1}{2} g^{\mu \nu} \partial_{\mu} \phi \partial_{\nu} \phi\left(1-f \frac{\sigma^{2}}{\mu^{2}}\right)-\frac{1}{2} g^{\mu \nu} \partial_{\mu} \sigma \partial_{\nu} \sigma-V(\phi) .
$$

Let us again consider large-field inflation with $V(\phi) \propto \phi^{n}$, where $n=\mathcal{O}(1)$. Here $\sigma$ receives an effective mass from the inflaton kinetic term, therefore instead of (7) the following approximation is assumed,

$$
3 H \dot{\sigma} \sim-f \dot{\phi}^{2} \frac{\sigma}{\mu^{2}} .
$$

Then (5) and 27) require the necessary conditions,

$$
\frac{M_{p}^{2}}{\phi^{2}} \ll 1, \quad\left|f \frac{\sigma^{2}}{\mu^{2}}\right| \ll 1, \quad\left|\frac{M_{p}^{2}}{\phi^{2}} f \frac{M_{p}^{2}}{\mu^{2}}\right| \ll 1,
$$

where the last condition is equivalent to $m_{\sigma}^{2} / H^{2} \ll 1$. Now, (5) and 27) are combined to yield

$$
\ln \frac{\sigma}{\sigma_{*}} \sim \frac{n}{3} f \frac{M_{p}^{2}}{\mu^{2}} \ln \frac{\phi}{\phi_{*}} .
$$

Since now the effective mass of $\sigma$ is suppressed by the slow-roll parameter $\epsilon, \sigma$ tends to roll less compared to the previous section. Let us further assume

$$
\left|f \frac{M_{p}^{2}}{\mu^{2}}\right| \ll 1
$$


and study cases where the modulus field is fixed during inflation, i.e. $\sigma \sim$ const. Then from

$$
d \mathcal{N} \simeq-\frac{V d \phi}{M_{p}^{2} V^{\prime}}\left\{1-f \frac{\sigma^{2}}{\mu^{2}}+\mathcal{O}(\epsilon, \eta)\right\}
$$

we arrive at

$$
\frac{\partial \mathcal{N}}{\partial \sigma} \sim-\frac{\phi^{2}-\phi_{f}^{2}}{n M_{p}^{2}} f \frac{\sigma}{\mu^{2}} \sim-2 \mathcal{N} f \frac{\sigma}{\mu^{2}},
$$

where $\mathcal{N}$ is the number of e-foldings obtained between $\phi$ and $\phi_{f}$, cf. (18). Since $\sigma \sim$ const., its correction to the inflaton dynamics stays constant all through inflation and the resulting curvature perturbation becomes proportional to $\mathcal{N}$. This means that the conversion of the entropy to curvature perturbations happen more at larger $\phi$, where more e-foldings are generated.

Note that (32) can also be obtained as a $\sigma \rightarrow \sigma_{f}$ limit of $(20)$ with $m=0$. (Remember that when $m=0$, the $\sigma$-correction to the inflaton potential had no effect on $\delta \mathcal{N}$, cf. (13).) However we emphasize that in 20 , $\sigma \sim \sigma_{f}$ suppresses the curvature perturbation to $|\partial \mathcal{N} / \partial \sigma| \ll\left|\sigma / M_{p}^{2}\right|$. (Especially for $m=0,\left|g \frac{\phi^{2}}{\mu^{2}}\right| \ll 1$ was the condition for $\sigma \sim \sigma_{f}$.) Whereas in the present case 32, , one need not require $\left|f \frac{\phi^{2}}{\mu^{2}}\right| \ll 1$ for $\sigma$ staying constant, therefore $|\partial \mathcal{N} / \partial \sigma|$ can become larger than $\left|\sigma / M_{p}^{2}\right|$. This is nothing but stating that $\sigma$ tends to roll less in the present case due to the small effective mass induced by the inflaton kinetic term.

Curvature perturbations from $\delta \phi$ is the same as in the previous section, i.e. (19). Then the ratio between contributions from $\delta \phi(19)$ and $\delta \sigma(32)$ is now

$$
\frac{\partial \mathcal{N} / \partial \sigma}{\partial \mathcal{N} / \partial \phi} \sim-f \frac{\sigma \phi}{\mu^{2}}
$$

where we have used $\phi^{2} \gg \phi_{f}^{2}=\mathcal{O}(1) \times M_{p}^{2}$. For example, $\phi \sim 10 M_{p}, \sigma \sim M_{p}$, and $f \frac{M_{p}^{2}}{\mu^{2}} \sim \frac{1}{10}$ make curvature perturbations from $\delta \phi$ and $\delta \sigma$ comparable.

Before continuing, let us look into additional $\delta \mathcal{N}$ produced at the end of and after inflation, which was briefly discussed in the previous section.

Where inflation ends is shifted as $\phi_{f}=\phi_{f_{0}}\left(1+\mathcal{O}\left(f \frac{\sigma^{2}}{\mu^{2}}\right)\right)$ where $\phi_{f_{0}}$ is the value in the absence of the $\sigma$-correction, hence modulation of $\phi_{f}$ is of order (note that when $\sigma$ stays constant, $\delta \phi$ does not modulate $\phi_{f}$ )

$$
\frac{\delta \phi_{f}}{\phi_{f}}=\mathcal{O}\left(f \frac{\sigma}{\mu^{2}}\right) \delta \sigma
$$

This modulates 18 and yields

$$
\delta \mathcal{N}_{f} \sim-\frac{\phi_{f}}{n M_{p}^{2}} \delta \phi_{f}=-\frac{\phi_{f}^{2}}{n M_{p}^{2}} \mathcal{O}\left(f \frac{\sigma}{\mu^{2}}\right) \delta \sigma .
$$

Since $\phi^{2} \gg \phi_{f}^{2}$, this contribution at the end of inflation is small compared to 32 which is mostly generated at large $\phi$.

Further $\delta \mathcal{N}$ can be produced after inflation, due to the fact that the energy density is not equal on the surface where inflation ends in field space. This effect can be estimated by computing the modulation of the potential energy at the end of inflation,

$$
\delta \mathcal{N}_{c}=\frac{1}{4} \frac{\delta V_{f}}{V_{f}}=\frac{n}{4} \frac{\delta \phi_{f}}{\phi_{f}}=\mathcal{O}\left(f \frac{\sigma}{\mu^{2}}\right) \delta \sigma,
$$

where we have assumed that the universe becomes radiation dominated right after inflation ends. One sees that this effect is of the same order as (35), hence can also be ignored.

Focusing on the curvature perturbation sourced by $\delta \sigma \sqrt{32}$ ), its spectral index (note especially that $\mathcal{P}_{\zeta} \propto \mathcal{N}^{2}$ induces $\left.n_{s}-1 \supset-2 / \mathcal{N}\right)$ is

$$
n_{s}-1 \sim 2\left(-\frac{1}{\mathcal{N}}+\frac{\dot{H}}{H^{2}}\right) \sim-n(n+4) \frac{M_{p}^{2}}{\phi^{2}}
$$


and the non-Gaussianity parameter defined as in 25 is

$$
f_{\mathrm{NL}} \sim-\frac{1}{4 \mathcal{N}} \frac{\mu^{2}}{f \sigma^{2}} \sim-\frac{n}{2} \frac{M_{p}^{2}}{\phi^{2}} \frac{\mu^{2}}{f \sigma^{2}}
$$

In this subsection we have mainly focused on the case where $\sigma$ stays constant during inflation, but extending the calculations to rolling $\sigma$ is straightforward. Basically, as one can guess easily, $\partial \mathcal{N} / \partial \sigma$ is reduced (amplified) for $\sigma$ with large positive (negative) $m_{\sigma}^{2}$. However, in contrast to what we have studied in the previous section, one finds that even in cases where $\sigma$ rolls towards zero, the curvature perturbations sourced by sub-Planckian $\sigma$ can exceed that from super-Planckian $\phi$.

\section{B. $\sigma$-corrections with different minima}

In actual cases, it may well be that the $\sigma$-corrections show up both in the inflaton kinetic term and potential, but with different minima. Then after redefining $\sigma$ so that the minimum of the potential correction is zero (and also absorbing extra constant terms to $\phi$ ), we arrive at the Lagrangian

$$
\frac{\mathcal{L}}{\sqrt{-g}}=-\frac{1}{2} g^{\mu \nu} \partial_{\mu} \phi \partial_{\nu} \phi\left(1-h \frac{\sigma}{\mu}-f \frac{\sigma^{2}}{\mu^{2}}\right)-\frac{1}{2} g^{\mu \nu} \partial_{\mu} \sigma \partial_{\nu} \sigma-V(\phi)\left(1+g \frac{\sigma^{2}}{\mu^{2}}\right) .
$$

Now the linear $\sigma$-correction (i.e. the term with $h$ ) gives additional modulation. However for large $\sigma$, i.e. $|\sigma| \gg\left|\frac{h}{f} \mu\right|$, the $h$-term becomes negligible compared to the $f$-term. Then the difference in minima can be ignored and the previous discussions are applied.

On the other hand when $\sigma$ is small as $|\sigma| \ll\left|\epsilon \frac{h}{g} \mu\right|$, then now the $g$-term becomes negligible, i.e., the leading backreaction to $\sigma$, and modulation to the inflaton dynamics are both sourced by the $\sigma$-correction in the inflaton kinetic term. Thus we recover the situation in Subsection III A.

A behavior unique to (39) arises when $\sigma$ is in the intermediate range so that the $\sigma$-dynamics is controlled by the $g$-term, while the leading modulation to the inflaton dynamics is given by the linear $h$-term.

If $|g| \gtrsim|f|$, then the necessary conditions for (5) and

$$
3 H \dot{\sigma} \sim-2 g V \frac{\sigma}{\mu^{2}}
$$

to hold under the Lagrangian $(39)$ are that the absolute values of the following quantities to be smaller than one,

$$
\epsilon, \quad \eta, \quad h \frac{\sigma}{\mu}, \quad g \frac{\sigma^{2}}{\mu^{2}}, \quad g \frac{M_{p}^{2}}{\mu^{2}}, \quad \epsilon \frac{h}{g} \frac{\mu}{\sigma} .
$$

The last quantity is required to be small so that the $\sigma$-dynamics (i.e. the right hand side of (40)) is given by the $g$-term, not the $h$-term. (Some terms in (8) are absent here since we are considering the $m=0$ case in (1) with an additional linear $h$-term.) Then, computing the curvature perturbations from $\delta \sigma$ as in Section II] now we obtain an additional contribution from the $h$-term,

$$
\frac{\partial \mathcal{N}}{\partial \sigma} \supset \frac{\partial}{\partial \sigma} \int_{\phi}^{\phi_{f}} \frac{\widetilde{V} d \tilde{\phi}}{M_{p}^{2} \widetilde{V}^{\prime}} h \frac{\tilde{\sigma}}{\mu} \sim-\frac{h}{2 g} \frac{\mu}{M_{p}^{2}}\left(1-\frac{\sigma_{f}}{\sigma}\right)
$$

Note that this result holds for arbitrary inflaton potential $V(\phi)$. This contribution becomes dominant over that from the $f$-term: $\frac{\partial \mathcal{N}}{\partial \sigma} \supset-\frac{f}{2 g} \frac{\sigma}{M_{p}^{2}}\left\{1-\left(\frac{\sigma_{f}}{\sigma}\right)^{2}\right\}$ (this $m=0$ result from 20 is also valid for arbitrary $V(\phi)$ ) when $\left|\sigma+\sigma_{f}\right| \ll\left|\frac{h}{f} \mu\right|$. (Here, since $m=0$, the $g$-term does not modulate $d \mathcal{N}$ at its linear order $g \frac{\sigma^{2}}{\mu^{2}}$. However if $|g| \gg|f|$, then its higher order contributions may exceed the linear order contribution from the $f$-term.) Thus, $\sigma$ can source curvature perturbations in a way different from the previous cases in the $\left|\epsilon \frac{h}{g} \mu\right| \ll|\sigma| \ll\left|\frac{h}{f} \mu\right|$ region. 


\section{EXAMPLES: LIGHT FIELDS IN D-BRANE INFLATION MODELS}

For concrete realizations of the above discussions, let us study D-brane inflation models in string theory. Usually in this class of models the position of a D-brane plays the role of the inflaton, but its dynamics can be modulated by other physical degrees of freedom of the brane. In this section we focus on the extra spatial directions in the internal manifold, and KK modes (oscillation modes) of wrapped branes.

\section{A. Monodromy-driven inflation with wrapped D-branes}

When inflation is driven by D-branes wrapped on cycles of the internal geometry [18, 31, 32, KK modes in the wrapped direction(s) (i.e. oscillation modes) can modulate the zero mode dynamics. In this subsection we focus on the model proposed in [18 where monodromy elongates the wrapped cycle and yields large-field inflation. In such case the KK modes become more and more light as the cycle becomes large. ${ }^{6}$

In the paper [18, the authors consider ten-dimensional type IIA string theory compactified on an orientifold of a product of two nil three-manifolds [33. The nil three-manifold $\mathcal{N}_{3}$ has the geometry

$$
\begin{aligned}
\frac{d s_{\text {nil }}^{2}}{\alpha^{\prime}} & =L_{u_{1}}^{2} d u_{1}^{2}+L_{u_{2}}^{2} d u_{2}^{2}+L_{x}^{2}\left(d x+\frac{M}{2}\left[u_{1} d u_{2}-u_{2} d u_{1}\right]\right)^{2} \\
& =L_{u_{1}}^{2} d u_{1}^{2}+L_{u_{2}}^{2} d u_{2}^{2}+L_{x}^{2}\left(d x^{\prime}+M u_{1} d u_{2}\right)^{2}
\end{aligned}
$$

(where $x^{\prime}=x-\frac{M}{2} u_{1} u_{2}$ ) compactified by

$$
\begin{aligned}
\left(x, u_{1}, u_{2}\right) & \rightarrow\left(x+1, u_{1}, u_{2}\right), \\
\left(x, u_{1}, u_{2}\right) & \rightarrow\left(x-\frac{M}{2} u_{2}, u_{1}+1, u_{2}\right), \\
\left(x, u_{1}, u_{2}\right) & \rightarrow\left(x+\frac{M}{2} u_{1}, u_{1}, u_{2}+1\right) .
\end{aligned}
$$

Then assuming a D4-brane wrapped along the $u_{2}$ direction and moving along $u_{1}$ (with fixed $x^{\prime}$ ), its position in the $u_{1}$ direction can become the inflaton.

Let us now derive the inflaton action obtained in [18, but this time including the KK modes. We give in the appendix a general expression for the four-dimensional effective action obtained from a wrapped 4-brane. The formulae there are applied to the present case by $u_{1} \rightarrow r$ and $u_{2} \rightarrow \lambda / 2 \pi$. Henceforth, we set $u_{1}=r$ and use $r$ instead of $u_{1}$. Then for large $r$, i.e. $r \gg L_{u_{2}} / M L_{x}$, the relevant six-dimensional part of the metric takes the form (A1) with

$$
\begin{aligned}
& g_{r r}=\alpha^{\prime} L_{u_{1}}^{2} \equiv A^{2}, \\
& g_{\lambda \lambda}=\frac{1}{(2 \pi)^{2}} \alpha^{\prime} L_{x}^{2} M^{2} r^{2} \equiv B^{2} r^{2} .
\end{aligned}
$$

Then for zero $B_{2}$ field in the world volume directions, and vanishing world volume gauge field strength, one can compute the DBI (or simply the Nambu-Goto) action of the D4-brane of the form A2). After integrating out the wrapped direction, we obtain the four-dimensional effective action for the zero mode position of the brane with an infinite tower of KK modes. (For detailed derivation, see the appendix.) The zero mode $r_{0}$ and the KK modes $r_{n}$ $(n \neq 0)$ are almost canonically normalized by

$$
\phi \equiv \frac{2}{3} A\left(2 \pi p T_{4} B\right)^{1 / 2} r_{0}^{3 / 2}, \quad \psi_{n} \equiv A\left(2 \pi p T_{4} B\right)^{1 / 2} r_{0}^{1 / 2} r_{n} .
$$

\footnotetext{
${ }^{6}$ For a brane wrapped along a direction $\lambda_{1}$ with cycle length $l_{1}$, the elongation of the wrapped cycle can be understood as the monodromy allowing the brane to wrap multiple times $m$ along another direction $\lambda_{2}$ (with length $l_{2}$ ), while spaced evenly in the $\lambda_{1}$ direction. Therefore the mass of the KK modes is of order $m_{\mathrm{KK}} \sim 1 / m l_{2}$, cf. $n^{2} / p^{2}$ term in A5. Furthermore, when the spacing between the multiply wrapped brane becomes narrow, light open string modes can also emerge with mass $m_{\text {open }} \sim l_{1} / m \alpha^{\prime}$. However, we note that these open strings stretching between the wrapped brane are basically heavier than the KK modes as long as the length scales of the internal space are larger than the string scale, i.e. $l_{1} l_{2}>\alpha^{\prime}$. We thank Hirosi Ooguri for helpful discussions on this point.
} 
Considering small oscillations around the zero mode position, i.e. $\left|r_{n}\right| \ll r_{0}$, or $\left|\psi_{n}\right| \ll \phi$, the action up to two derivatives and quadratic order in $\left(r_{n}, \partial_{\mu} r_{n}\right)$ is obtained as follows,

$$
\begin{aligned}
S=\int d^{4} x \sqrt{-g^{(4)}}[ & -\frac{1}{2}(\partial \phi)^{2}\left(1-\frac{2}{9} \frac{A^{2}}{B^{2}} \sum_{n \neq 0} \frac{n^{2}}{p^{2}} \frac{\left|\psi_{n}\right|^{2}}{\phi^{2}}-\frac{1}{3} \sum_{n \neq 0} \frac{\left|\psi_{n}\right|^{2}}{\phi^{2}}\right)-\frac{1}{2} \sum_{n \neq 0}\left(\partial \psi_{n}\right)\left(\partial \bar{\psi}_{n}\right) \\
& \left.-\left(\frac{3 \pi p T_{4} B}{A}\right)^{2 / 3} \phi^{2 / 3}\left(1+\frac{2}{9} \frac{A^{2}}{B^{2}} \sum_{n \neq 0} \frac{n^{2}}{p^{2}} \frac{\left|\psi_{n}\right|^{2}}{\phi^{2}}\right)-\frac{1}{3} \sum_{n \neq 0} \frac{\psi_{n}}{\phi}(\partial \phi)\left(\partial \bar{\psi}_{n}\right)\right],
\end{aligned}
$$

where $p$ is the winding number. Dropping the KK modes $\psi_{n}$, one recovers the inflaton action obtained in [18, with potential $V(\phi) \propto \phi^{2 / 3}$. Here, the $\psi_{n}$-correction to the inflaton kinetic term can be understood as nonzero $\psi_{n}$ increasing the wrapped volume, therefore correcting the canonical normalization (46). The correction to the inflaton potential is due to the brane tension damping the oscillation, which gives $\psi_{n}$ effective mass which becomes lighter for larger $\phi$, i.e. for longer cycle. However, the KK modes also receive tachyonic instability from their coupling to the zero mode motion (note that during inflation $a^{3} \sum \frac{\psi_{n}}{\phi} \dot{\phi} \dot{\bar{\psi}}_{n} \simeq-\frac{3}{2} a^{3} H \frac{\dot{\phi}}{\phi} \sum\left|\psi_{n}\right|^{2}+$ (total derivatives)). This effective tachyonic mass can be seen explicitly if instead of $\phi$ we introduce

$$
\varphi \equiv \frac{2}{3} A\left(2 \pi p T_{4} B\right)^{1 / 2} r_{0}^{3 / 2}\left(1+\frac{3}{8} \sum_{n \neq 0} \frac{\left|r_{n}\right|^{2}}{r_{0}^{2}}\right),
$$

for which the action becomes

$$
\begin{aligned}
S=\int d^{4} x \sqrt{-g^{(4)}}[ & -\frac{1}{2}(\partial \varphi)^{2}\left(1-\frac{2}{9} \frac{A^{2}}{B^{2}} \sum_{n \neq 0} \frac{n^{2}}{p^{2}} \frac{\left|\psi_{n}\right|^{2}}{\varphi^{2}}\right)-\frac{1}{2} \sum_{n \neq 0}\left(\partial \psi_{n}\right)\left(\partial \bar{\psi}_{n}\right) \\
& \left.-\left(\frac{3 \pi p T_{4} B}{A}\right)^{2 / 3} \varphi^{2 / 3}\left(1+\frac{2}{9} \frac{A^{2}}{B^{2}} \sum_{n \neq 0} \frac{n^{2}}{p^{2}} \frac{\left|\psi_{n}\right|^{2}}{\varphi^{2}}-\frac{1}{9} \sum_{n \neq 0} \frac{\left|\psi_{n}\right|^{2}}{\varphi^{2}}\right)\right] .
\end{aligned}
$$

Basically, if $A^{2} \ll B^{2}$ the lower KK modes obtain tachyonic instability. (One can image a cone which spreads out quickly away from its tip.)

We comment that, as was discussed in [18, the $\mathcal{N}_{3} \times \widetilde{\mathcal{N}}_{3}$ space also allows a variant inflaton action. Given a different configuration for the D4-brane (e.g. by wrapping the brane along the $u_{2}-\tilde{u}_{2}$ direction while moving it in a linear combination of $u_{1}+\tilde{u}_{1}$ and $u_{2}+\tilde{u}_{2}$ directions with $\left.u_{1}=\tilde{u}_{1}\right)$, a metric $g_{r r} \propto r^{2}, g_{\lambda \lambda} \propto r^{2}$ can be realized for large $r$, which gives an inflaton potential $V(\phi) \propto \phi^{2 / 5}$. One can check that in this case, the $\psi_{n}$-correction terms proportional to $\frac{n^{2}}{p^{2}}$ take the form $\frac{\left|\psi_{n}\right|^{2}}{\mu^{4 / 5} \phi^{6 / 5}}$.

Going back to the action 47 or 499 , the example values chosen in [18] for the parameters give $\frac{A^{2}}{B^{2}}=(2 \pi)^{2} \frac{L_{u_{1}}^{2}}{L_{x}^{2} M^{2}} \gtrsim$ $10^{4}$, so for winding number $p=\mathcal{O}(1)$ the tachyonic instability is negligible. There, since the CMB scale leaves the horizon at $\phi / M_{p}=\mathcal{O}(10)$ for $V(\phi) \propto \phi^{2 / 3}$, the effective mass of $\psi_{n}$ becomes heavier than the Hubble parameter, i.e. the last condition of (10) is not satisfied. However, since the parameters can be shifted in variants of the construction, let us give general discussions for cases where the metric takes the form (45).

We assume $\frac{A^{2}}{B^{2}} \frac{n^{2}}{p^{2}} \ll 10^{3}$, so that the effective mass of $\psi_{n}$ is lighter than the Hubble parameter when the CMB scale exits the horizon. Then, for $\frac{A^{2}}{B^{2}} \frac{n^{2}}{p^{2}} \gg 1$, the KK modes $\psi_{n}$ roll towards zero and from the discussions given in Section III curvature perturbations generated from fluctuations of $\psi_{n}$ is negligible compared to that from the inflaton $\phi$. Meanwhile, if $\frac{A^{2}}{B^{2}} \frac{n^{2}}{p^{2}} \ll 1$ the KK modes $\psi_{n}$ are tachyonic. Substituting $n=2 / 3, m=2, f=0$, and $g=-1 / 9$ to 20 , one finds the "amplification factor" (i.e. $\left.\left(\sigma_{f} / \sigma\right)^{2}\right)$ to be $\left(\phi_{f} / \phi\right)^{2 / 3}$, which is smaller than 10.

One can also check that when $\delta \phi$ sources the dominant contribution to the curvature perturbations, non-Gaussianity produced by the KK modes $\psi_{n}$ is negligible, i.e. $\left|\frac{\partial^{2} \mathcal{N}}{\partial \sigma^{2}}\left(\frac{\partial \mathcal{N}}{\partial \sigma}\right)^{2} /\left(\frac{\partial \mathcal{N}}{\partial \phi}\right)^{4}\right| \ll 1$, for both $\frac{A^{2}}{B^{2}} \frac{n^{2}}{p^{2}} \gg 1$ and $\ll 1$ cases as long as $\left|\psi_{n}\right| \ll|\phi|$ holds during inflation.

Therefore, for the metric 45 with $\frac{A^{2}}{B^{2}} \frac{n^{2}}{p^{2}} \gg 1$ or $\ll 1$, we conclude that unless the KK modes start off from Planckian or super-Planckian values, we can safely neglect such modes during inflation. However, one may also need to look into their cosmological implications after inflation, since even though they become heavy after (or towards 
the end of) inflation, they can carry on non-negligible oscillation amplitudes from the early times in the inflationary era when they were light.

Before ending this section, we note that given additional contributions to the inflaton action other than from the Nambu-Goto part of the DBI action, then the KK modes' corrections to the inflaton potential can be weakened while the corrections to the inflaton kinetic term remains effective. Especially for wrapped D-brane inflation models in warped throats [31, 32] where one expects various sources contributing to the inflaton potential, the cases studied in Section III may be realized and then one needs to take into account of the KK modes seriously.

\section{B. Warped D-brane inflation}

D3-brane inflation models in a warped throat geometry [19, 34, 40] admit light field corrections from angular directions. One may expect that a finite region of the throat is well modeled by a noncompact throat (such as a conifold) possessing angular isometries, plus corrections which break the isometries due to the throat being glued to a compact bulk. Then, given that such corrections are well suppressed by the warping, light angular directions can emerge in the regime deep into the throat. (Hence for an inflaton D3 moving towards the throat tip, light angular directions are expected to show up at later times in the inflationary era, which is in contrast to the previous subsection where the KK modes were lighter at earlier times. If inflation ends by brane annihilation, then one may not have to worry about the cosmological moduli problem for the light directions at all.) Usually the model is treated as a single field model of the radial position of the D3-brane, by fixing its angular positions at their local minima and integrating out the angular degrees of freedom. However, in cases where the angular directions become light, one may have to treat such directions as dynamical fields. ${ }^{7}$

In simple cases, the D3-brane's radial $r$ and periodic angular directions $\theta^{i}$ are canonically normalized to $\phi \propto r$ and $\sigma^{i} \propto r \theta^{i}$ (hence $\left.|\phi| \gtrsim\left|\sigma^{i}\right|\right)$. Then one expects an inflaton potential corrected by terms $\sigma^{i} / \phi$ multiplied by some powers of the warp factor. Furthermore, if inflation ends by D3- $\overline{\mathrm{D} 3}$ annihilation at the throat tip, then the $\overline{\mathrm{D} 3}$-brane's angular degrees of freedom $\tilde{\sigma}$ may also become light and give corrections of the form $\tilde{\sigma} / \mu$.

One can imagine that various contributions to the inflaton potential have different dependence on angular positions of D3/ $\overline{\mathrm{D} 3}$, giving an action highly more complicated than (1). However, from the discussions in the previous sections, we expect that the multi-field effects can become important if the D3/D3 starts off near a local maximum of the angular directions and rolls down during inflation. It would be interesting to examine the effects of angular directions in a concrete and computable setup of warped D3-brane inflation.

\section{CONCLUSIONS}

We have studied effects of light fields showing up as small corrections in the inflaton action. While minimally affecting the inflaton dynamics, such moduli can still leave imprints on cosmological observables through generating curvature perturbations. ${ }^{8}$ The fluctuations of the moduli are converted to curvature perturbations during inflation, due to patches of the universe taking different trajectories in field space. The basic picture is simple: for converging trajectories the transformation of entropy to adiabatic perturbations is suppressed at later times, and for diverging trajectories it is enhanced. The curvature perturbation produced by the moduli is especially amplified and can dominate over that from the inflaton when the moduli receive tachyonic backreaction from the inflaton potential, or when the main moduli corrections enter the inflaton kinetic term. In this paper we mainly focused on modulating large-field inflation with $V(\phi) \propto \phi^{n}$, but it would be interesting to perform a systematic study with more general inflaton potentials. The effects of moduli can be simply stated as modulating the relation between $d \mathcal{N}$ and $d \phi$, hence they can operate in basically any inflation model. Modulating models beyond the slow-roll ones may have distinct features and is worthy of investigation. (For e.g., rapid-roll 20 22, and DBI inflation models 23, 24. Both are well-motivated by the warped D-brane inflation setup, which provides many candidate light fields as was discussed in Section [V],

\footnotetext{
7 Generating curvature perturbations at the end of inflation (by shifting $\phi_{f}$ ) with light angular directions were investigated in 41 , 42 . Also, in [43, the two-field dynamics of the D3's radial position and the volume modulus was studied. For DBI inflation with multiple fields, see e.g. [44-46].

8 While in this paper we have studied effects of light fields minimally affecting the inflaton dynamics, heavy fields which considerably alter the inflaton dynamics may also have interesting consequences. For example, 47] demonstrated cases where such heavy field effects generate equilateral non-Gaussianity.
} 
Our study has a range of applications. As in the D-brane inflation models we have studied in the paper, it is important to examine the (in)validity of integrating out extra degrees of freedom in inflation models consisting of multiple fields. Also, when discussing cosmological scenarios where the presence of light fields are inevitable or required (e.g. curvaton, modulated reheating scenario), one needs to look into the fields' effects not just after inflation, but also during inflation. In this work, for some simple cases we provided conditions under which the moduli can be ignored. On the other hand we also exhibited cases where the light moduli dominantly source the curvature perturbations. The mechanism of generating curvature perturbations from light moduli instead of the inflaton may open up new possibilities for inflation model building.

\section{Acknowledgments}

We are grateful to Damien Easson, Masahiro Kawasaki, Tatsuma Nishioka, Hirosi Ooguri, and David Wands for useful conversations. The work of T.K. was supported by Grant-in-Aid for JSPS Fellows No. 21.8966. The work of S.M. was supported in part by Grant-in-Aid for Young Scientists (B) No. 17740134, Grant-in-Aid for Creative Scientific Research No. 19GS0219, Grant-in-Aid for Scientific Research on Innovative Areas No. 21111006, Grant-inAid for Scientific Research (C) No. 21540278, and the Mitsubishi Foundation. This work was supported by World Premier International Research Center Initiative (WPI Initiative), MEXT, Japan.

\section{Appendix A: Effective Action of a Wrapped 4-Brane}

Here we give a general expression for the four-dimensional effective action derived from the Nambu-Goto action of a 4-brane wrapping a 1-cycle. We consider the 4-brane to be stretching along the external space $x^{\mu}(\mu=0,1,2,3)$, while wrapping the $\lambda$ direction with winding number $p$ and moving along the $r$ direction in the internal space. The six-dimensional part of the metric which is relevant for us is assumed to take the following form,

$$
d s^{2}=g_{\mu \nu}^{(4)}(x) d x^{\mu} d x^{\nu}+g_{r r}(r) d r^{2}+g_{\lambda \lambda}(r) d \lambda^{2},
$$

with the $\lambda$ direction compactified by $\lambda \simeq \lambda+2 \pi$. Taking the brane coordinates to coincide with $x^{\mu}$ and $\lambda$, the Nambu-Goto action of the 4-brane is

$$
S=-T_{4} \int d^{4} x \int_{0}^{2 \pi p} d \lambda \sqrt{-\operatorname{det}\left(G_{M N} \partial_{m} X^{M} \partial_{n} X^{N}\right)},
$$

(where $T_{4}$ is the 4 -brane tension) with

$$
\operatorname{det}\left(G_{M N} \partial_{m} X^{M} \partial_{n} X^{N}\right)=g^{(4)}\left\{g_{\lambda \lambda}+g_{\lambda \lambda} g_{r r} g^{(4) \mu \nu} \partial_{\mu} r \partial_{\nu} r+g_{r r}\left(\partial_{\lambda} r\right)^{2}\right\},
$$

where $g^{(4)}=\operatorname{det}\left(g_{\mu \nu}^{(4)}\right)$.

Now let us expand the radial position of the 4-brane as

$$
r\left(x^{\mu}, \lambda\right)=\sum_{n=-\infty}^{\infty} r_{n}\left(x^{\mu}\right) e^{i \frac{n}{p} \lambda}
$$

where $\bar{r}_{n}=r_{-n}$. Upon expanding the action A2 up to two $x^{\mu}$-derivatives and quadratic order in $\left(r_{n}, \partial_{\mu} r_{n}\right)$, and then integrating out the $\lambda$ direction, we arrive at

$$
\begin{aligned}
& S \simeq-2 \pi p T_{4} \int d^{4} x \sqrt{-g^{(4)}} \sqrt{g_{\lambda \lambda}} \\
& \times\left[\frac{1}{2} g_{r r} g^{(4) \mu \nu} \partial_{\mu} r_{0} \partial_{\nu} r_{0}\left\{1+\frac{1}{2} \sum_{n \neq 0}\left|r_{n}\right|^{2}\left(\frac{1}{2} \frac{g_{\lambda \lambda}^{\prime \prime}}{g_{\lambda \lambda}}-\frac{1}{4}\left(\frac{g_{\lambda \lambda}^{\prime}}{g_{\lambda \lambda}}\right)^{2}+\frac{g_{r r}^{\prime} g_{\lambda \lambda}^{\prime}}{g_{r r} g_{\lambda \lambda}}+\frac{g_{r r}^{\prime \prime}}{g_{r r}}-\frac{n^{2}}{p^{2}} \frac{g_{r r}}{g_{\lambda \lambda}}\right)\right\}\right. \\
&+\frac{1}{2} g_{r r} \sum_{n \neq 0} g^{(4) \mu \nu} \partial_{\mu} r_{n} \partial_{\nu} \bar{r}_{n}+\left(\frac{1}{2} \frac{g_{\lambda \lambda}^{\prime}}{g_{\lambda \lambda}}+\frac{g_{r r}^{\prime}}{g_{r r}}\right) g_{r r} \sum_{n \neq 0} r_{n} g^{(4) \mu \nu} \partial_{\mu} r_{0} \partial_{\nu} \bar{r}_{n} \\
&\left.+1+\frac{1}{2} \sum_{n \neq 0}\left|r_{n}\right|^{2}\left(\frac{1}{2} \frac{g_{\lambda \lambda}^{\prime \prime}}{g_{\lambda \lambda}}-\frac{1}{4}\left(\frac{g_{\lambda \lambda}^{\prime}}{g_{\lambda \lambda}}\right)^{2}+\frac{n^{2}}{p^{2}} \frac{g_{r r}}{g_{\lambda \lambda}}\right)\right],
\end{aligned}
$$


where $\sum_{n \neq 0} \equiv \sum_{n=-\infty, n \neq 0}^{\infty}$. The primes denote derivatives, and $g_{r r}, g_{\lambda \lambda}$ are functions of the zero mode $r_{0}\left(x^{\mu}\right)$.

[1] A. A. Starobinsky, Phys. Lett. B 91, 99 (1980).

[2] K. Sato, Mon. Not. Roy. Astron. Soc. 195, 467 (1981).

[3] A. H. Guth, Phys. Rev. D 23, 347 (1981).

[4] G. D. Coughlan, W. Fischler, E. W. Kolb, S. Raby and G. G. Ross, Phys. Lett. B 131, 59 (1983).

[5] T. Banks, D. B. Kaplan and A. E. Nelson, Phys. Rev. D 49, 779 (1994) arXiv:hep-ph/9308292.

[6] B. de Carlos, J. A. Casas, F. Quevedo and E. Roulet, Phys. Lett. B 318, 447 (1993) arXiv:hep-ph/9308325.

[7] A. D. Linde and V. F. Mukhanov, Phys. Rev. D 56, 535 (1997) arXiv:astro-ph/9610219.

[8] K. Enqvist and M. S. Sloth, Nucl. Phys. B 626, 395 (2002) arXiv:hep-ph/0109214].

[9] D. H. Lyth and D. Wands, Phys. Lett. B 524, 5 (2002) arXiv:hep-ph/0110002.

[10] T. Moroi and T. Takahashi, Phys. Lett. B 522, 215 (2001) [Erratum-ibid. B 539, 303 (2002)] arXiv:hep-ph/0110096].

[11] G. Dvali, A. Gruzinov and M. Zaldarriaga, Phys. Rev. D 69, 023505 (2004) arXiv:astro-ph/0303591.

[12] L. Kofman, arXiv:astro-ph/0303614.

[13] D. Polarski and A. A. Starobinsky, Nucl. Phys. B 385, 623 (1992).

[14] J. Garcia-Bellido and D. Wands, Phys. Rev. D 53, 5437 (1996) arXiv:astro-ph/9511029.

[15] C. Gordon, D. Wands, B. A. Bassett and R. Maartens, Phys. Rev. D 63, 023506 (2001) arXiv:astro-ph/0009131.

[16] S. Groot Nibbelink and B. J. W. van Tent, Class. Quant. Grav. 19, 613 (2002) arXiv:hep-ph/0107272.

[17] M. Sasaki, Prog. Theor. Phys. 120, 159 (2008) arXiv:0805.0974 [astro-ph]].

[18] E. Silverstein and A. Westphal, Phys. Rev. D 78, 106003 (2008) arXiv:0803.3085 [hep-th]].

[19] S. Kachru, R. Kallosh, A. D. Linde, J. M. Maldacena, L. P. McAllister and S. P. Trivedi, JCAP 0310, 013 (2003) arXiv:hep-th/0308055.

[20] A. D. Linde, JHEP 0111, 052 (2001) arXiv:hep-th/0110195.

[21] L. Kofman and S. Mukohyama, Phys. Rev. D 77, 043519 (2008) arXiv:0709.1952 [hep-th]].

[22] T. Kobayashi, S. Mukohyama and B. A. Powell, JCAP 0909, 023 (2009) arXiv:0905.1752 [astro-ph.CO]].

[23] E. Silverstein and D. Tong, Phys. Rev. D 70, 103505 (2004) arXiv:hep-th/0310221.

[24] M. Alishahiha, E. Silverstein and D. Tong, Phys. Rev. D 70, 123505 (2004) arXiv:hep-th/0404084].

[25] A. A. Starobinsky, JETP Lett. 42, 152 (1985) [Pisma Zh. Eksp. Teor. Fiz. 42, 124 (1985)].

[26] M. Sasaki and E. D. Stewart, Prog. Theor. Phys. 95, 71 (1996) arXiv:astro-ph/9507001.

[27] D. Wands, K. A. Malik, D. H. Lyth and A. R. Liddle, Phys. Rev. D 62, 043527 (2000) arXiv:astro-ph/0003278.

[28] D. H. Lyth, K. A. Malik and M. Sasaki, JCAP 0505, 004 (2005) arXiv:astro-ph/0411220.

[29] D. H. Lyth, JCAP 0511, 006 (2005) arXiv:astro-ph/0510443.

[30] L. Alabidi and D. Lyth, JCAP 0608, 006 (2006) arXiv:astro-ph/0604569.

[31] T. Kobayashi, S. Mukohyama and S. Kinoshita, JCAP 0801, 028 (2008) arXiv:0708.4285 [hep-th]].

[32] M. Becker, L. Leblond and S. E. Shandera, Phys. Rev. D 76, 123516 (2007) arXiv:0709.1170 [hep-th]].

[33] E. Silverstein, Phys. Rev. D 77, 106006 (2008) arXiv:0712.1196 [hep-th]].

[34] D. Baumann, A. Dymarsky, I. R. Klebanov, J. M. Maldacena, L. P. McAllister and A. Murugan, JHEP 0611, 031 (2006) arXiv:hep-th/0607050.

[35] O. DeWolfe, L. McAllister, G. Shiu and B. Underwood, JHEP 0709, 121 (2007) arXiv:hep-th/0703088.

[36] D. Baumann, A. Dymarsky, I. R. Klebanov, L. McAllister and P. J. Steinhardt, Phys. Rev. Lett. 99, 141601 (2007) arXiv:0705.3837 [hep-th]].

[37] A. Krause and E. Pajer, JCAP 0807, 023 (2008) arXiv:0705.4682 [hep-th]].

[38] D. Baumann, A. Dymarsky, I. R. Klebanov and L. McAllister, JCAP 0801, 024 (2008) arXiv:0706.0360 [hep-th]].

[39] D. Baumann, A. Dymarsky, S. Kachru, I. R. Klebanov and L. McAllister, JHEP 0903, 093 (2009) arXiv:0808.2811 [hep-th]].

[40] D. Baumann, A. Dymarsky, S. Kachru, I. R. Klebanov and L. McAllister, arXiv:1001.5028 [hep-th].

[41] D. H. Lyth and A. Riotto, Phys. Rev. Lett. 97, 121301 (2006) arXiv:astro-ph/0607326.

[42] H. Y. Chen, J. O. Gong and G. Shiu, JHEP 0809, 011 (2008) arXiv:0807.1927 [hep-th]].

[43] S. Panda, M. Sami and S. Tsujikawa, Phys. Rev. D 76, 103512 (2007) arXiv:0707.2848 [hep-th]].

[44] D. Langlois, S. Renaux-Petel, D. A. Steer and T. Tanaka, Phys. Rev. Lett. 101, 061301 (2008) arXiv:0804.3139 [hep-th]].

[45] D. Langlois, S. Renaux-Petel, D. A. Steer and T. Tanaka, Phys. Rev. D 78, 063523 (2008) [arXiv:0806.0336 [hep-th]].

[46] F. Arroja, S. Mizuno and K. Koyama, JCAP 0808, 015 (2008) arXiv:0806.0619 [astro-ph]].

[47] A. J. Tolley and M. Wyman, Phys. Rev. D 81, 043502 (2010) arXiv:0910.1853 [hep-th]]. 